\title{
The laboratory diagnosis of gonorrhoea and syphilis infection
}

\author{
Edward L Chan, PhD, FCCM, Director of Clinical Microbiology, Department of Pathology, Baptist Medical Center-Montclair, \\ Birmingham, AL, USA; Margaret A Kingston, MRCP, DFFP, Consultant in Genitourinary Medicine, Manchester Centre for \\ Sexual Health and Manchester Children's University Hospitals NHS Trust, Manchester Royal Infirmary, Manchester, UK; \\ Elizabeth M Carlin, DFFP, FRCP, Consultant in Genitourinary Medicine, Department of Genitourinary Medicine, Nottingham \\ City Hospital NHS Trust, Nottingham, UK
}

\author{
Correspondence: Dr Edward L Chan, Department of Pathology, Baptist Medical Center-Montclair, Birmingham, \\ AL 35213-1984, USA. E-mail: Edward.Chan@BHSALA.com
}

(Accepted 19 October 2003)

Journal of Family Planning and Reproductive Health Care 2004; 30(2): 126-127

\section{Background: epidemiology and clinical features}

Following the decline in incidence of gonorrhoea and syphilis in the mid-1980s, there has been a resurgence in cases of both infections during the late 1990s. Since 1994 there has been an ongoing rise in cases of gonorrhoea, with a $9 \%$ increase between 2001 and 2002 in the UK. Men who have sex with men (MSM) and black ethnic groups, mainly in urban areas, are disproportionately affected. ${ }^{1}$ There is also evidence of increased antimicrobial resistance in worldwide Neisseria gonorrhoeae isolates, especially to ciprofloxacin and azithromycin. ${ }^{2}$ A recent study in the UK demonstrated a prevalence of ciprofloxacin resistance in $N$. gonorrhoeae of $9.8 \%$, a two- to three-fold rise from 2001 to 2002, irrespective of overseas sexual contact, sex or area of residence. ${ }^{3}$ In women, infection is asymptomatic in about $50 \%$ of cases, but if symptomatic the commonest symptom is of altered vaginal discharge. In men a purulent urethral discharge is commonly present. Pharyngeal and rectal infection occurs in both sexes and is frequently asymptomatic.

Syphilis is caused by the spirochaete bacterium, Treponema pallidum. It is systemic from the outset, infectious in the early stages, and if untreated has a chronic relapsing course. Clinical manifestations of the infection are highly variable and from Victorian times, when up to one in nine Europeans were affected, it has been known as 'the great pretender'. It is transmitted by sexual contact or vertically from mother to child during pregnancy. Initially, a painless, indurated ulcer known as a 'chancre' develops at the site of inoculation. This resolves and may be followed by an erythematous psoriatic rash and orogenital ulcers, mucous patches or condylomata lata (condylomatous lesions in the genital area), which are also transient and often accompanied by systemic symptoms. Untreated the infection becomes latent and neurological, cardiovascular and other sequelae may occur up to 30 years after initial infection in up to $40 \%$ of untreated individuals. $^{4}$ If syphilis develops during pregnancy, intrauterine death, stillbirth or congenital syphilis frequently occur. From 1998 to 2002, an 803\% increase in cases of early syphilis was observed in the UK, mainly comprised of localised outbreaks in MSM. ${ }^{1}$ Outbreaks have been reported from a variety of locations but the two largest and ongoing outbreaks are in London and Manchester in the UK.

\section{Diagnosis of $N$. gonorrhoeae infection \\ Specimen collection}

For women a swab should be taken from the endocervix (rotating it there for a few seconds) and the urethra. Rectal samples should also be taken depending on the sexual history, if a partner has gonococcal infection, or if she is symptomatic at the site. Pharyngeal samples should be taken if she has practised receptive orogenital sex. For men a urethral swab plus a rectal and/or pharyngeal swab should be taken as indicated by the sexual history and symptoms. The swab should be rolled onto a glass slide to be air-dried and Gram-stained, and then either inserted in transport media or inoculated directly to an agar plate which is suitable for gonococcal culture.

\begin{abstract}
Microscopy
In genitourinary medicine (GUM) clinics microscopy is performed on site as a near-patient test, allowing rapid diagnosis. Under light microscopy $(\times 1000)$ the typical intracellular Gram-negative diplococci are observed in polymorphonuclear leucocytes. For male urethral samples this has a sensitivity of $89-96 \%$, whereas for female endocervical smears it is only $31-51 \% .^{5}$ Microscopy is not appropriate for pharyngeal samples. Although of lower sensitivity, microscopy of rectal smears is of use (particularly in symptomatic cases) to allow rapid diagnosis.
\end{abstract}

\section{Culture}

Culture is a readily available, cheap, sensitive and specific test that also allows sensitivity testing to be performed. Sensitivity testing is essential for the early detection of changes in sensitivity patterns and enables appropriate changes in antibiotic therapy to be initiated. Culture is the diagnostic method of choice in most cases including medico-legal cases such as child abuse and sexual assault. Results are generally available within 72 hours. Selective and non-selective lysed blood agars, typically based on a modified New York City media, are often used since a small proportion of gonococci may be sensitive to vancomycin in selective media. ${ }^{6}$

\section{Nucleic acid detection and amplification tests}

Several assays are available including tests able to detect $N$. gonorrhoeae in urine, vaginal, urethral and cervical samples. 7,8 Such assays are highly sensitive (95\%) and specific $(99 \%)$ in symptomatic patients from areas of high prevalence. For this reason, in low prevalence areas positive cases require confirmation with a second assay.

The Gen-probe assay uses a labelled DNA probe to detect $N$. gonorrhoeae ribosomal RNA in specimens. It is not a nucleic acid amplification test as it does not amplify the target. Sensitivity is around $85 \%$, and with a confirmation assay specificity approaches $98 \%$.

An important limitation of these tests is that they do not allow sensitivity testing which, as discussed earlier, is of increasing importance. Therefore, culture confirmation and sensitivity testing in those patients identified with 
gonorrhoea by nucleic acid assay tests is essential. If culture confirmation is not possible then it is crucial to perform a test-of-cure following treatment, ideally using a culture method.

\section{Diagnosis of syphilis}

Demonstrating the presence of $\mathrm{T}$. pallidum

T. pallidum cannot be cultured in vitro, but the presence of the bacterium in the infectious lesions of primary or secondary syphilis may be demonstrated. Dark-field microscopy involves sampling the lesion's exudates and immediate dark-field microscopy demonstrates the motile treponemes. This requires expertise most often available in GUM clinics and does allow immediate near-patient diagnosis. It is, however, unsuitable for oral and rectal lesions since visual differentiation between oral and bowel spirochaetes is not possible. An alternative method, namely fluorescent microscopy, involves air-drying the slide and staining it with rabbit fluorescent anti-T. pallidum antibody and then examining the slide for spirochaetes. As this method is specific for T. pallidum it is suitable for oral and rectal lesions, but requires expertise and is not widely available. Polymerase chain reaction can be performed on swabs taken from possible syphilitic lesions and placed in transport media. This is a sensitive and specific test for $T$. pallidum, and has recently been shown to be an important adjunct to treponemal serology in diagnosing infectious syphilis during the current UK outbreaks, ${ }^{9}$ although it is not yet widely available.

\section{Serological tests for syphilis}

Following infection with $T$. pallidum, antibodies are produced to many of the treponemal antigens. The intensity of reaction reflects clinical symptoms, and by the time symptoms are evident all but $10-15 \%$ of those individuals with early primary syphilis will have immunoglobulin $G$ (IgG) and $\mathrm{M}$ (IgM) antibodies. Antibody responses decrease with time from infection and following treatment, allowing the response to treatment to be monitored. The antibody response to infection is not protective, and reinfection may occur with a corresponding increase in antibody titres.

Treponemal tests detect antibody specific to $T$. pallidum. They include enzyme immunoassay (EIA), $T$. pallidum haemagglutination assay (TPHA), T. pallidum particle agglutination test (TPPA) and fluorescent treponemal antibody with absorption test (FTA-ABS). EIA assays may test for anti-IgG alone or IgG and IgM in combination. They have high specificity, sensitivity and are suited to automation, and so are increasingly being used as the initial screening test. If the EIA is positive it is confirmed with another treponemal test, usually the TPPA or TPHA since both tests are very sensitive and specific, particularly the TPPA. A non-treponemal test is then performed to assist in diagnosing the stage of infection.

Non-treponemal tests include the rapid plasma regain test (RPR) and venereal diseases research laboratory test (VDRL). These tests detect non-specific treponemal antibody, and quantification of the response allows assessment of stage of infection and response to treatment as discussed above. A high RPR or VDRL titre is indicative of recent, active infection and a fall in titre four-fold following treatment is considered a good response to therapy. ${ }^{4}$ False-positive results may occur, for example, during pregnancy, febrile illnesses and in those with autoimmune conditions. This limits the use of nontreponemal tests as initial screening tests and underlines the fact that no serological test should be used for diagnosis in isolation. In addition, when very high levels of nontreponemal antibody are present a false-negative result, the 'prozone phenomenon', may occur. If this situation is suspected clinically then the sample should be diluted and retested, and this is one reason why sending full clinical details to the laboratory with the sample is crucial.

\section{Summary}

Following a decline in prevalence during the 1980s and early 1990s, gonorrhoea and syphilis infections are once again posing a threat to public health. In addition, the antibiotic sensitivity pattern for gonorrhoea appears to have changed with an increased prevalence of resistance. Both syphilis and gonorrhoea appear to disproportionately affect MSM and black ethnic minorities, and are concentrated in urban areas. Their diagnosis requires microbiological tests to be performed appropriately, and a rapid diagnosis can often be provided in GUM clinics using near-patient microscopy. Early diagnosis and effective, rapid treatment is crucial in limiting the morbidity for the affected individual and the public health risks resulting from the spread of infection.

Statements on funding and competing interests

Funding. None identified.

Competing interests. None identified.

References

1 Gonorrhoea: epidemiological data. http://www.hpa.org.uk/infections/ topics.

2 Fluoroquinolone-resistance in Neisseria gonorrhoeae, Hawaii 1999, and decreased susceptibility to azithromycin in $N$. gonorrhoeae, Missouri 1999. MMWR Morb Mortal Wkly Rep 2000; 49: 833-837.

3 Fenton KA, Ison, C, Johnson AP, et al. Ciprofloxacin resistance in Neisseria gonorrhoeae in England and Wales in 2002. Lancet 2003; 361: $1867-1869$

4 Clinical Effectiveness Group (Association of Genitourinary Medicine and Medical Society for the Study of Venereal Diseases). National guideline for the management of early syphilis. http:// www.agum.org.uk/guidelines.htm.

5 Gooheart ME, Ogden AJ, Zaidi AA, et al. Factors affecting the performance of smear and culture tests for the detection of Neisseria gonorrhoeae. Sex Trans Dis 1982; 4: 63-69.

6 Mirrett S, Reller LB, Knapp JS. Neisseria gonorrhoeae strain inhibited by vancomycin in selective media and correlation with auxotype. J Clin Microbiol 1981; 14: 94-99.

7 Stary A, Ching SF, Teodorowicz, et al. Comparison of ligase chain reaction and culture for detection of Neisseria gonorrhoeae in genital and extragenital specimens. J Clin Microbiol 1997; 35: 239-242.

8 Hook EW, Ching SF, Stephens J, et al. Diagnosis of Neisseria gonorrhoeae infections in women by using the ligase chain reaction on patient-obtained vaginal swabs. J Clin Microbiol 1997; 35: 239-242.

9 Palmer HM, Higgins SP, Herring AJ, et al. Use of PCR in the diagnosis of early syphilis in the United Kingdom. Sex Transm Infect 2003; 79: 479-483

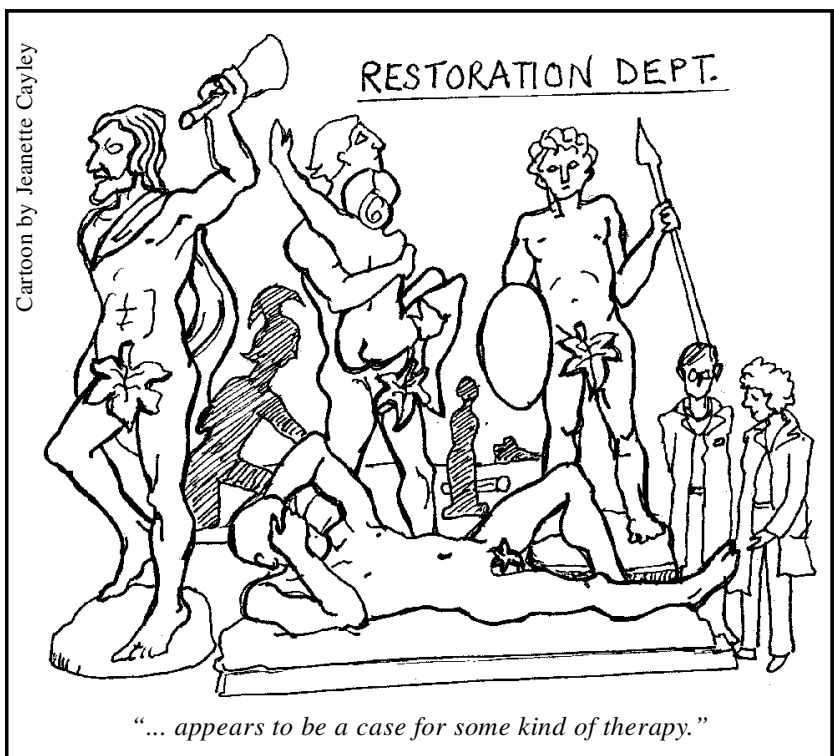

